\title{
Editorial
}

\section{Numerical and Analytical Methods for Variational Inequalities and Related Problems with Applications}

\section{Zhenyu Huang, ${ }^{1}$ Ram N. Mohapatra, ${ }^{2}$ Muhammad Aslam Noor, ${ }^{3}$ Hong-Kun $X_{u}{ }^{4}$ and Qingzhi Yang ${ }^{5}$}

\author{
${ }^{1}$ Department of Mathematics, Nanjing University, Nanjing 210093, China \\ ${ }^{2}$ Department of Mathematics, University of Central Florida, Orlando, FL 32816, USA \\ ${ }^{3}$ Mathematics Department, COMSATS Institute of Information Technology, Islamabad, Pakistan \\ ${ }^{4}$ Department of Applied Mathematics, National Sun Yat-Sen University, Kaohsiung 804, Taiwan \\ ${ }^{5}$ School of Mathematics and LPMC, Nankai University, Tianjin 300071, China
}

Correspondence should be addressed to Zhenyu Huang, zhenyu@nju.edu.cn

Received 23 October 2012; Accepted 23 October 2012

Copyright (C) 2012 Zhenyu Huang et al. This is an open access article distributed under the Creative Commons Attribution License, which permits unrestricted use, distribution, and reproduction in any medium, provided the original work is properly cited.

The study of variational inequalities and related problems with applications constitutes a rich topic of intensive research efforts within the latest 50 years. Variational inequality theory, which was introduced by Stampacchia in 1964, has emerged as a fascinating branch of mathematical and engineering sciences with a wide range of applications in industry, finance, economics, ecology, social, regional, pure, and applied sciences. The corresponding iterative methods have witnessed great progress in recent years to handle problems in optimization problems, inverse problems, and differential equations.

We received 61 research papers in the research fields. This special issue includes 23 high-quality peer-reviewed papers. The aim of this special issue has been to present the latest and generalized coverage of the fundamental and constructive ideas, concepts, and important issues in the accepted original research articles as well as comprehensive review articles stimulating the continuing efforts to numerical analysis for variational inequality problems and fixed-point problems with applications.

In the fascinating paper by S. Saewan and P. Kumam, the existence and convergence analysis of the solutions of system of mixed variational inequalities in Banach spaces are given by using the generalized projection operator. N. Wairojjana and P. Kumam provide several general iterative methods for finding the solutions to variational inequalities. Modified block iterative methods are presented by K. Wattanawitoon and P. Kumam for asymptotically relatively nonexpansive mappings and for systems of generalized mixed equilibriums. In the setting of Hilbert spaces, several distinguished researchers, 
R. Wangkeeree and P. Preechasilp; K. Wattanawitoon and P. Kumam; A. Wang, are working successfully on finding the common solutions to various equilibrium problems, variational inequalities, and fixed point problems. For the more general Banach spaces, the corresponding problems are studied by distinguished people such as J.-H. Zhu et al. in their original independent work. Seven published papers work on the related differential equations and applications. In the interesting paper by M. A. Noor et al., the relationship between differential equations and general variational inequalities is established with numerical methods. These wonderful results in the studies of differential equations are given independently by S. Chai and Y. Zou; Y. Sun; W.-X. Zhou and H.-Z. Liu; J. Gohet al.; M. Delkhosh and M. Delkhosh; H. Yang and F. Jiang. Eight published papers have good studies on providing solutions to the complementarity problems by $\mathrm{H}$. Sun and Y. Wang; properties for multivalued nonexpansive mappings in $R$-trees by K. Samanmit and B. Panyanak; iterative methods for strict pesudocontractions by B.-C. Deng et al.; 1norm regularization method for split feasibility problems by $\mathrm{S}$. He and $\mathrm{W}$. Zhu; gradientprojection algorithms for averaged mappings by M. Tian and M.-M. Li; implicit and explicit algorithms for asymptotically nonexpansive mappings by $\mathrm{M}$. Wen et al.; well-posedness for hemivariational inequalities by S. Lv et al.; improved initial values for polynomial zeros by S. Saidanlu et al.

\title{
Acknowledgments
}

The Editors would like to express their deepest gratitude to the authors for their fascinating and interesting contributions as well as to the staff and the editorial office of the journal for the great and invaluable support. The Editors would like also to express their greatest appreciation to more than 200 reviewers for their important time and valuable suggestions/comments to make the special issue successful with highly qualified published papers.

\author{
Zhenyu Huang \\ Ram N. Mohapatra \\ Muhammad Aslam Noor \\ Hong-Kun Xu \\ Qingzhi Yang
}




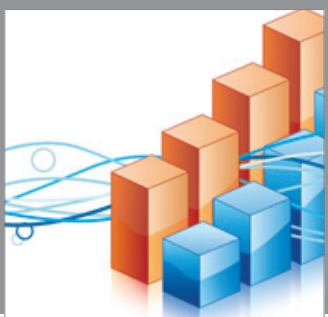

Advances in

Operations Research

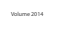

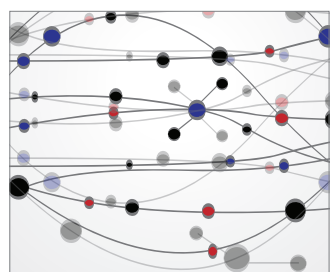

\section{The Scientific} World Journal
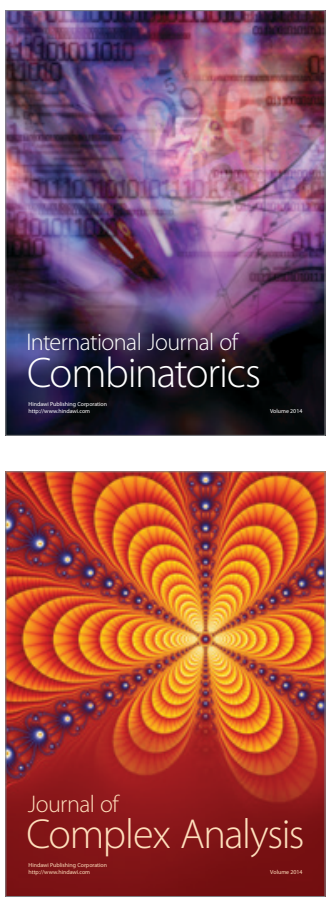

International Journal of

Mathematics and

Mathematical

Sciences
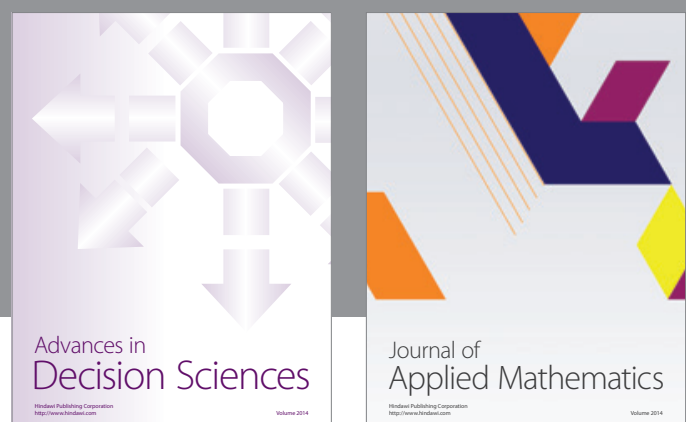

Journal of

Applied Mathematics
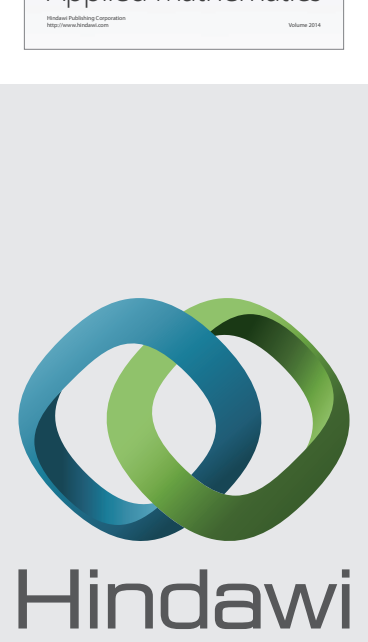

Submit your manuscripts at http://www.hindawi.com
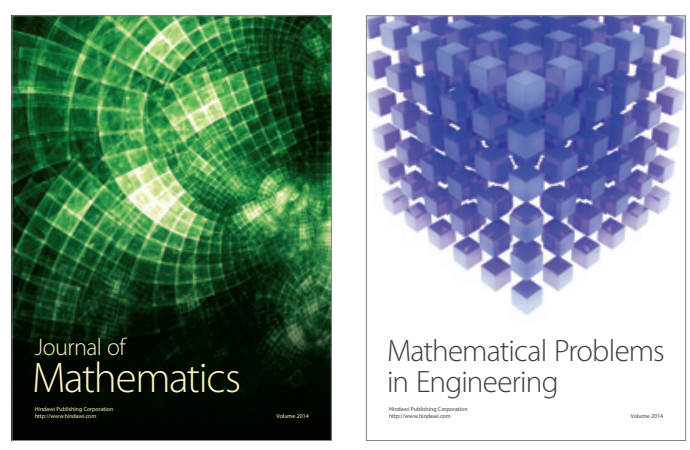

Mathematical Problems in Engineering
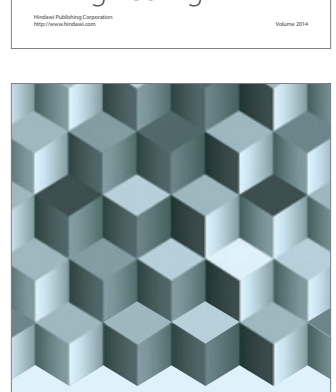

Journal of

Function Spaces
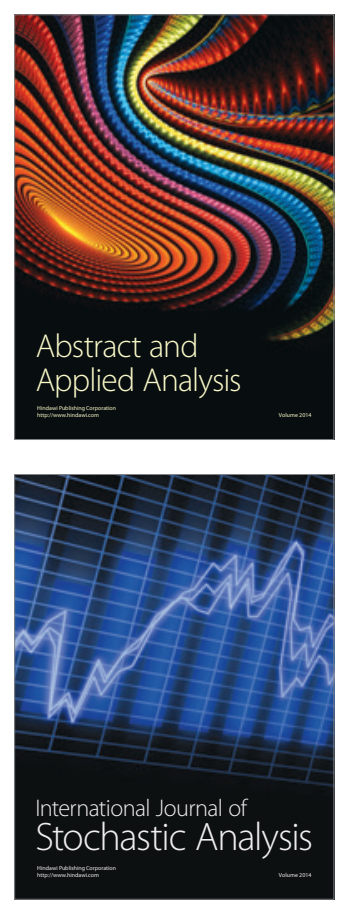

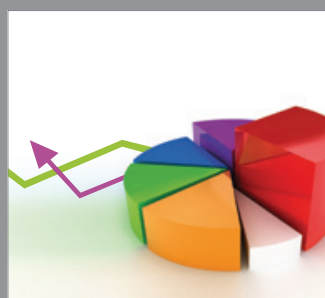

ournal of

Probability and Statistics

Promensencen
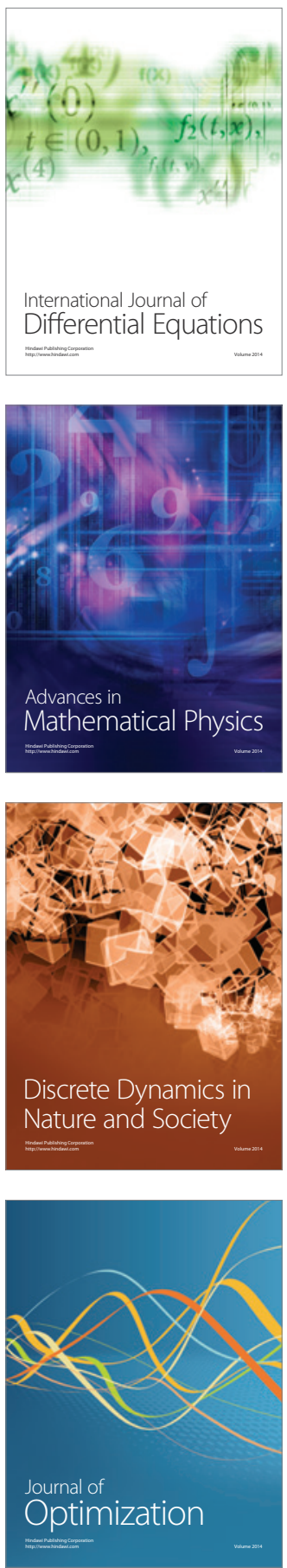